\title{
Malignant Thymus Neoplasm
}

National Cancer Institute

\section{Source}

National Cancer Institute. Malignant Thymus Neoplasm. NCI Thesaurus. Code C4962.

A primary or metastatic malignant neoplasm involving the thymus. This category

includes malignant thymomas, thymic lymphomas, primary thymic carcinomas, and

metastatic carcinomas from other anatomic sites. 